\title{
Physical Training Model Design and Evaluation under Informatization
}

\author{
Shi Feng ${ }^{1, a}$ \\ ${ }^{1}$ The Security Department of Xingtai Medical College,Xingtai,Hebei,054000,China \\ asfylj1980@163.com
}

Keywords: Physical training, model, agent, system

\begin{abstract}
This paper, based on characteristics of physical training management, deeply studies DSS system structure on the basis of Multi-Agent and designs the Multi-Agent system structure that is suitable for physical training management. DSS of physical training management can formulate corresponding training planning for groups or individuals, provide decision support for two aspects of physical fitness assessment and physical training method, and offer scientific theoretical foundation to formulate plans.
\end{abstract}

\section{Introduction}

With the enhancement of our economic development level and continuous improvement of social life, people also gradually pay attention to health ${ }^{[1,2,3]}$. "Health First" and "Lifelong Sport" have already developed into the theme of physical exercise in new era. As the every-increasing physical exercise, innovation of apparatus is also received new requirement. Obviously, traditional apparatus can't satisfy development requirement of new era any more. The tendency of sports application in the era is to innovate and reform apparatus ${ }^{[5]}$. For many years, physical education in our colleges and universities has given priority to learn western modern athletics, resulting in devices of site apparatus lay particular stress on competitive sports and bring about problems of site and apparatus. Though, various colleges and universities make a huge investment, site and apparatus become important factors of restricting college physical education. At present, many domestic colleges and universities have established all kinds of sports venues and stadiums. It can satisfy requirement of physical education and college teaching evaluation in a certain period of time, but there are still some problems, such as lower use ratio of school stadiums, higher maintenance cost that can't be used for physical education, site and apparatus sufficiently, and new wasting of resources.

By studying and analyzing physical exercise, training and management for current college students, and combining computer artificial intelligence with current analytical investigation on physical exercise and decision-making method of training management, the paper finds out a kind of decision support method that conforms to modern physical exercise management theory, enhance physical fitness of college students and sports competition level of colleges. This paper studies a kind of DSS architecture based on Multi-Agent, designs a kind of Multi-Agent architecture that is suitable for physical training management, conducts specific implementation on various parts, and finally obtains DSS of physical training management based on Multi-Agent. The DSS of physical training management based on Multi-Agent can formulate corresponding training plans for groups and individuals, provide decision support for two aspects of physical fitness evaluation and physical training method, and offer scientific theoretical foundation to formulate plans.

\section{Physical Training Management Model Design Based on Agent}

Agent is a kind of computing entity or procedure that can sense environmental changes, make corresponding respondes positively and autonomously implement for the sake of realizing specific targets. Generally speaking, a Multi-Agent system composes of multiple Agents and cooperates with the agreemnt between Agents to complete and unify entire targets. Every Agent in the system is a stand-alone software entity and decides to operate collaterally for respectively decisive behaviors. It can be kept in a computer, or can be distributed to the network constituted by multiple computers to 
execute different missions. Agent can sense modules to reflect real world and abstract environmental message to a certain extent. According to types of information, sensing modules are delivered to different processing modules through abstract information. By combining with Agent, characteristics of Multi-Agent and DSS's features of physical training management, the DSS framework based on Multi-Agent is shold in Figure 1. Here, four kinds of Agents are defined as follows:

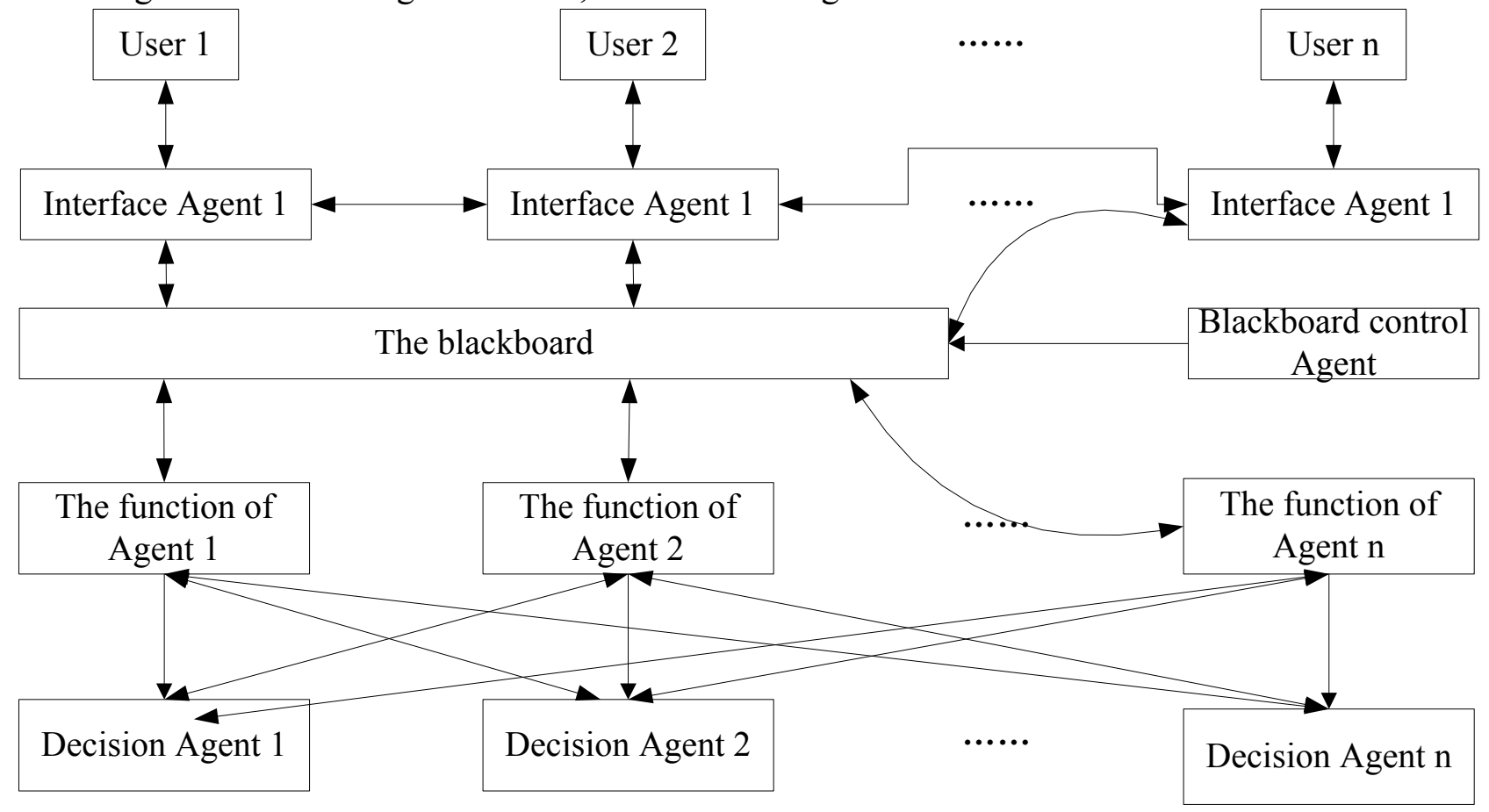

Figure 1 System Functional Chart

According to characteristics of problem solving and man-machine interaction, the paper expands basic Agent structure chart and obtains structure chart of interface Agent, as shown in Figure 2. Interface Agent composes of knowledge base, model base, communication module and inference engine, etc. Knowledge base stores some knowledge about user preference, interface organization mode and interface functional integration, etc., aspects. Model base stores basic objects of human-computer interface. User model is stored in model base by aiming at user habits and domain knowledge, reorganizing basic objects of machine learning and constantly adjusting by inference engine. In addition, there is interface graphical control, objects of if-then action expression, and objects of ask-if action expression, etc. The communication module is responsible for the interaction between interface Agent and blackboard, delivers decision-making requirement and data to decision users, and receives decision-making results passed by blackboard. Inference engine is the core of interface Agent and also is a link to connect with other parts in interface Agent. Each part in interface Agent can realize mutual interaction through inference engine. Primary work of inference engine is to combine with knowledge base, model base and user history to upgrade knowledge base and model base constantly, make human-computer interface become more scientific, reasonable and humanized, and ultimately achieve the goal of intelligent user interface. 


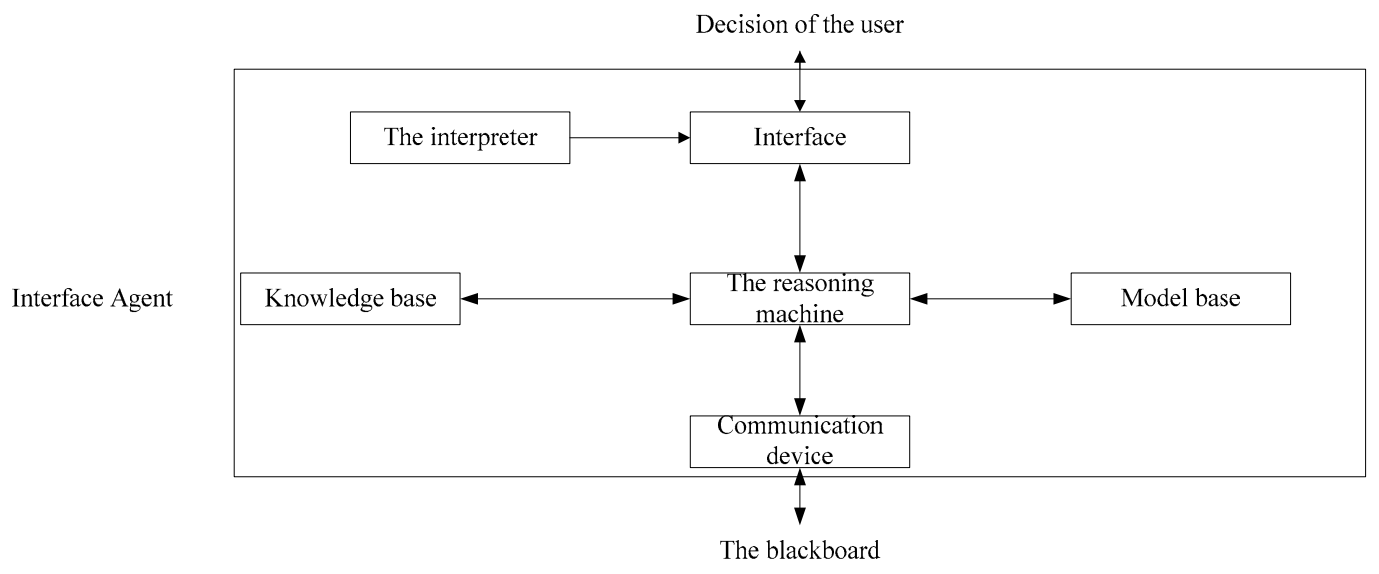

Figure 2 Structure Chart of Interface Agent

Blackboard control of Agent composes of inference engine, analyzer, executive module and learning machine, etc. The function of Agent also monitors the blackboard, matches with activated condition of decision-making Agent and level information of blackboard, and delivers information on the blackboard to corresponding decision of Agent. The function of Agent has an activated condition list to read blackboard for each decision of Agent. When changing information on the blackboard, corresponding related activated condition list should be checked. If it matches, it should be analyzed further. Decision directly activates corresponding decision of Agent to read blackboard information to complete corresponding task or engage in other operations, such as provision of report and explanation, etc. After the decision of Agent receives information, it should analyze firstly, and then conducts fuzzy matching with knowledge in knowledge base. If it can look for high matching knowledge, corresponding knowledge will be used for produce decision. If it only can match partly, matched knowledge will be regarded as condition, apply reasoning mechanism and rules in knowledge base to continue to match until it can produce decision or have no available knowledge. If there is no available knowledge, it should explain it correspondingly. Rules and knowledge in knowledge base will be added and upgraded constantly with the problem solving.

\section{Realization and Task Distribution Mechanism of Agent under Physical Training Model}

\section{Realization of Agent}

It mainly composes of three parts, including communication module, knowledge reasoning module and executive module. Communication module is responsible for receiving messages from the outside or other Agents, transfers it into the form that can be understood by Agent. Correspondingly, it should deliver messages to the outside or other Agents. Knowledge reasoning module includes property, method and behavior rule of Agent and upgrades automatically. They can provide factual basis for independent activities. Executive module is specific decision part in Agent. It makes decisions in line with property, method and behavior of knowledge reasoning module, and also needs to assist knowledge reasoning unit to upgrade knowledge.

PublieelassBase_Agent

\{publiestatiestring[]agents:, //Other Agent name lists of Agent publiestatie

stringAgentID://Name of Agent

protectedstaticAttribute[]attributes://Agent Related property

protectedstaticRule[]rules:// condition-action rule

publieBase_Agent()//Constructor

$\{\ldots \ldots . . .$.

ProteetedvoidCommunieate(Messmessage)

Communication function receives, preserves and reads messages related to Agent, is responsible for delivery message to other Agent//or blackboard, receives message delivered by other Agents or blackboard and transfers it to the form understood by this l'/Agent. 
PublicvoidExeeute(Attribute[]attributes, Rule[]rules, Messmessage)

Executive function makes corresponding decisions in line with property, rule and communication function of Agent. Realization process of executive function is also a reasoning process.

PublievoidGetRules(Rule[]rules, KBasekb)

//Obtain rules from knowledge base and save in ules.

PublievoidUPdate(Attribute[]attributes, KBasekb)

//Updated function upgrades property of Agent and rules in knowledge base in accordance with executive rules of decision function.

Now, parts of identifiers in the above-mentioned codes are explained as follows: Attribute is property, Rule is regulation, Mess is message and KBase is knowledge base.

In order to realize information interaction between multiple Agents, each Agent must abide by unified information interactive format, so message pattern of blackboard pattern is defined, as shown in Table 1.

Table 1 Message Format of Blackboard

\begin{tabular}{|c|l|c|c|c|c|}
\hline $\begin{array}{c}\text { Message of } \\
\text { identifier }\end{array}$ & $\begin{array}{l}\text { Origin } \\
\text { identifier of } \\
\text { Agent }\end{array}$ & $\begin{array}{c}\text { Targeted } \\
\text { identifier of } \\
\text { Agent }\end{array}$ & Permission & Life cycle & $\begin{array}{c}\text { Message } \\
\text { content }\end{array}$ \\
\hline
\end{tabular}

Message of identifier is the number of message in Agent discussion area. Origin identifier of Agent refers to mark number of delivering message of Agent. Targeted identifier of Agent refers to accept the mark number of receiving message. Permission means the jurisdiction to message operation, valuing as follows: ::read-only permission. $d$ is permission of deleting. Life cycle is the limit to standing time of read-only permission on the blackboard. It decreases progressively in line with Fibonacci sequence and as time goes on. When the count is reduced to zero, control unit should delete this message. In this system, interactive pattern based on between Agents of blackboard is shown in Figure 3.

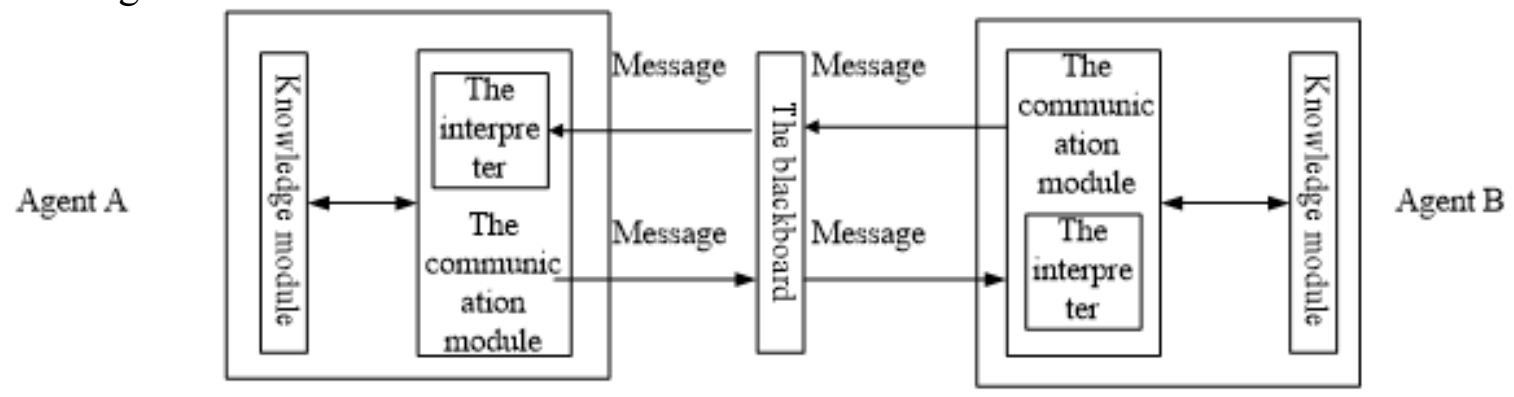

Figure 3 Interactive Pattern between Agents Based on Blackboard Model Structure

If Agent A wants to communicate with Agent B, first of all, it should deliver message to the blackboard through communication module. After blackboard receives requirement of Agent A, it will deliver message to Agent B and waits for its response. After Agent B receives message delivered by blackboard, it should determine whether it should accept requirement of Agent A in line with current situation, and then "accept" or "refuse". Result should be feedback to the blackboard. Ultimately, blackboard returns to Agent A.

\section{Multi-Agent Task Distribution Mechanism and Reasoning Mechanism}

Generally speaking, every Agent only has a part of Agent's function list in MAS system. Given that $\mathrm{A}, \mathrm{B}, \mathrm{C}, \mathrm{D}, \mathrm{E}$ and $\mathrm{F}$ are Agents in MAS system, while possible functional list stored by them many be indicated by matrix table, as shown in Figure 4. 


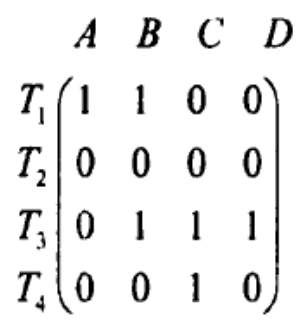

Agent A

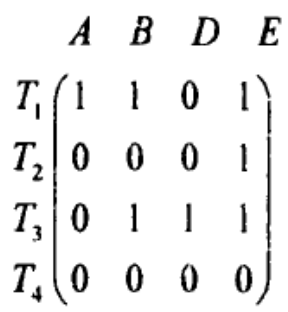

Agent B

\begin{tabular}{|c|c|c|}
\hline$T_{1}(1$ & 0 & \\
\hline \begin{tabular}{l|l}
$T_{2}$ & 0
\end{tabular} & 0 & 0 \\
\hline$T_{3}: 1$ & & 1 \\
\hline$T_{4}(0$ & & \\
\hline
\end{tabular}

Agent C

Figure 4 Search Matrix of Acquaintances

Functional lists of Agent A, Agent B and Agent C only include functions known by Agent. o means that Agent has the ability to perform. 1 means that Agent has the ability to perform this task. When Agent A accepts task T2. Though it has no ability to perform task, it knows that Agent B has the ability to perform through function table matrix. Therefore, it can make Agent B to accept requirement of Agent A to perform task through communication negotiation of Agent B. When Agent in function table has no ability to perform task, Agent A requires acquaintance Agent to use Agent A to look for solutions of task. For example, through Agent B, it can check whether Agent E can perform task until Agent in MAS system agree to accept this task. Or if the entire network has no response, it indicates that task can't be completed. For instance, when Agent A accepts task TZ, it not only has no ability to perform task, but also all Agents in Agent A function table have no ability to perform. Therefore, Agent A asks acquaintance Agent B in function table to check whether Agent can perform task TZ. The results find that Agent E has the ability to deal with this task, so Agent E of communication negotiation makes it accept this task.

In this system, the search strategy adopted by reference engine belongs to breadth-first search. The adoptive reasoning direction is forward reasoning way. The work flow diagram is shown in Figure 5. In data of Figure, first of all, user input conducts reasoning truth. In other words, about planning objects, it searches for the entire rule base, finds out partial matching or complete matching rules and store them into intermediate result set, gradually scan every rule concentrated by intermediate result, and delete unmatched rules from concentrated intermediate result. In this way, when intermediate result set is null value, it can obtain required conclusion. 


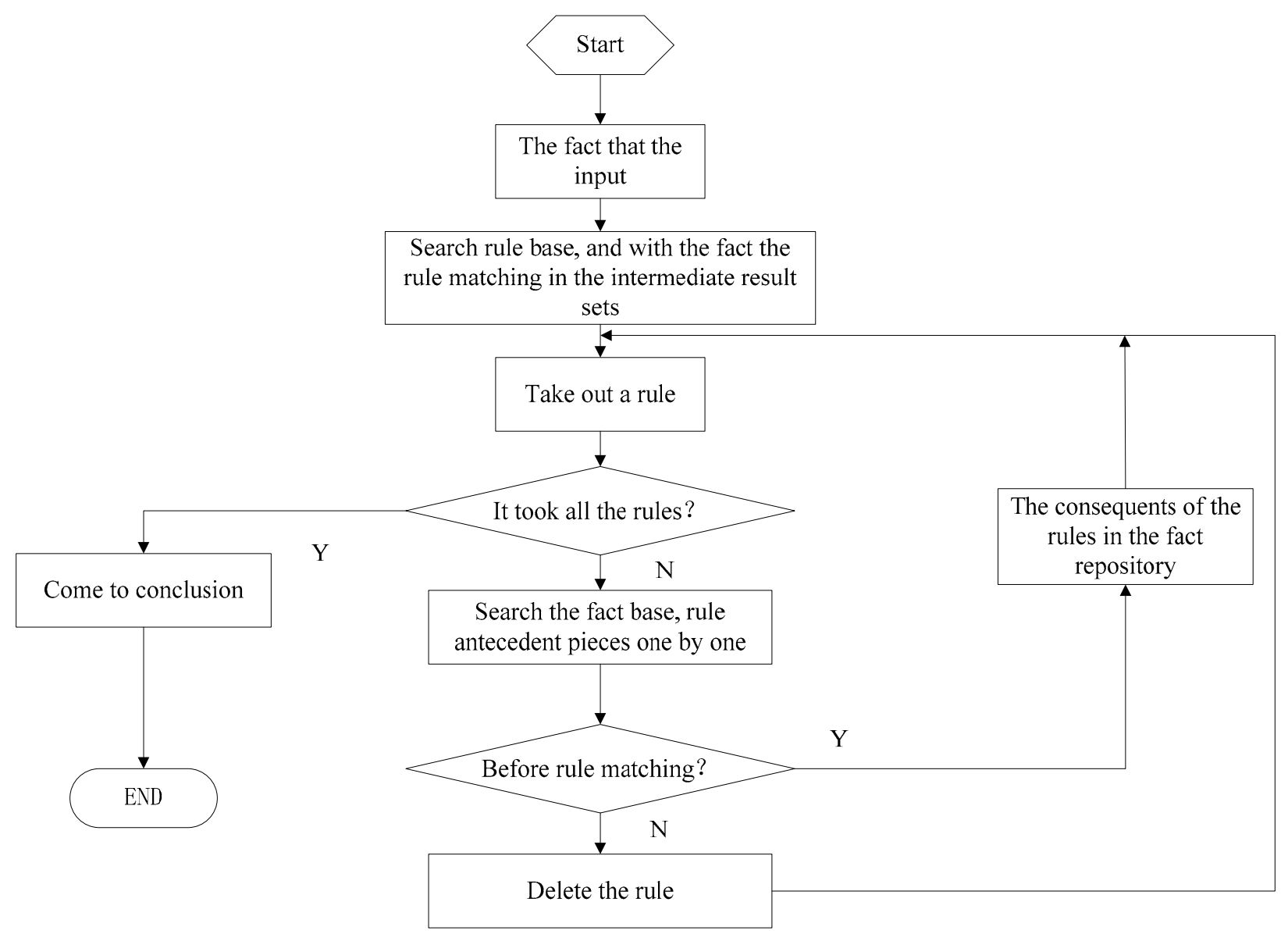

Figure 5 Workflow of Inference Engine

\section{Physical Training Model Empirical Analysis Based on Agent}

This topic adopts C\# and ASP.NET to develop. Because background database of this system uses SQLSERVER 20085, it adopts sqlclient pattern. M-Apriori is improved Apriori alogirthm and is used for frequent item set. Algorithm- $\mathrm{R}$ is association rule to produce algorithm. It searches for frequent item set and obtains association rule set in line with algorithm M-Apriori. Users apply assigned minimum support (min-suP) and minimum confidence coefficient min-conf to interact with M-Aprior and Algorithm-R, respectively, and explain and evaluate interaction of association rules on mining results.

Figure 6 uses the above-mentioned mining model to excavate hobbies of student movement. The total affairs are 10000 . The minimum support is $7 \%$. The minimum confidence coefficient is $27 \%$. Ultimately, it digs out 9 rules, remaining [8\%, 27\%]. It means that in all students' sport hobbies, $8 \%$ of students like swimming and table tennis. In students that like swimming, $27 \%$ of students like table tennis simultaneously. 


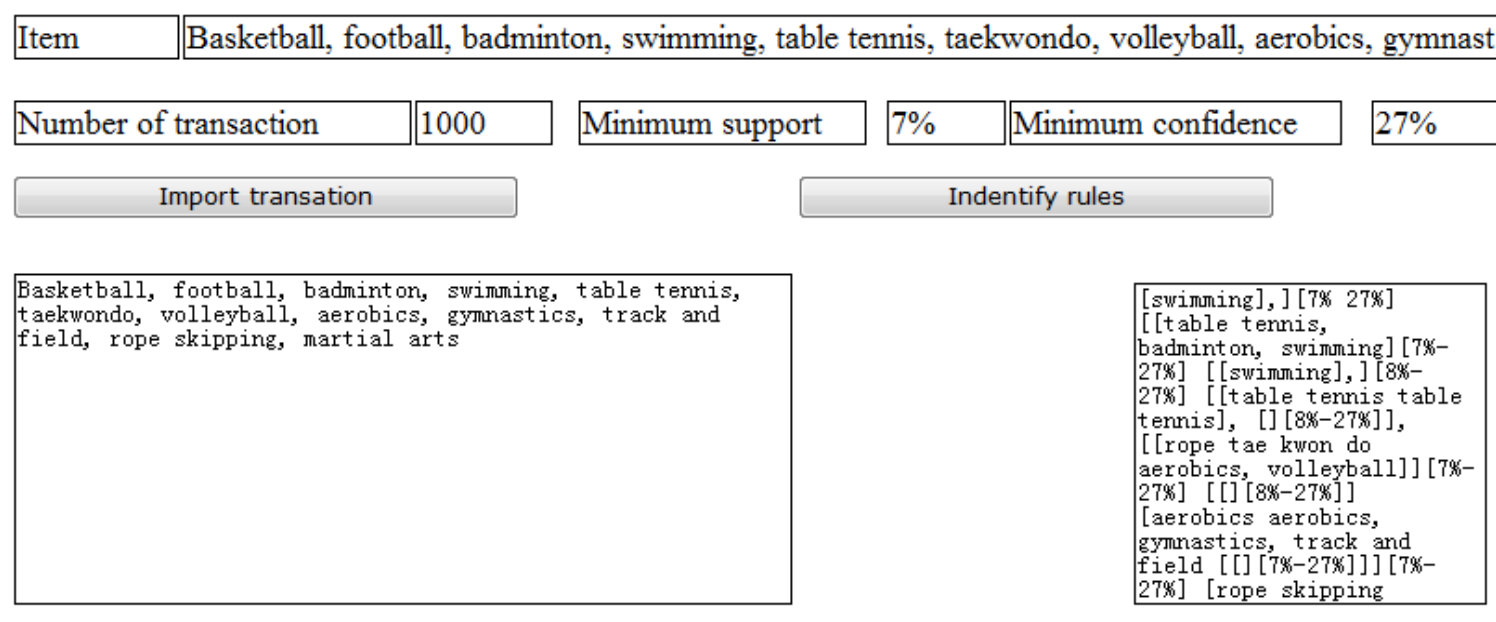

Figure 6 Selection of Students' Training Association Mining

\section{Summary}

Intelligence is inevitable tendency of computer application. No matter it is industrial control of computer, or commercial software for civil use, it requires increasingly higher intelligent factors. Multi-Agent technology is applied to intelligent decision support system, and realizes the intelligent knowledge system that publicizes and distributes closed and isolated knowledge system. Under dynamic environment, each Agent solves problems in the way of mutual negotiation, coordination and real time. This paper proposes several judgment criteria, which only reflects physical fitness of a person to some extent. Meanwhile, respective weighting of each standard is also subject to study deeply. This needs to the participation of experts in physical education, and also uses data mining technology to confirm. For judgment criteria of physical training project, it also needs to improve and also needs guidance of experts in physical training.

\section{References}

[1] Guo Xianyu, Thinking on Continuous Development of Amateur Sports, Journal of Shanghai University of Sport, 2001, Vol, 25, p137-139

[2] Fan Xiaojun, Analysis on Physical Amateur Training of Primary Schools, Postgraduate Dissertation Journal of Shanxi Normal University Physical Culture Institute, 2008, Vol, 23, p117-119

[3] Liu Zhihong, Study on School Physical Teaching Evaluation System Construction and Maneuverability, Hebei Normal University, 2007, p52-59

[4] Han Lingling, Development Research on Physical Education and Student Individual, Contemporary Sports Technology, 2011, Vol5, p53-55

[5] Tu Yanguo, Education Evaluation [M], Higher Education Press, 2007, Vol 3

[6] Yang Xudong, Development Research on Physical Education and Student Individual in Colleges, China Education Innovation Herald, 2011, Vol 8, p205

[7] Song Wei, Study on the Development Relationship between Physical Education and Urban Economy, Journal of Beijing University of Physical Education, 2004, Vol6, p736-738

[8] Hu Guoxiong and Wang Mudi, Case Study on Influence of Basic Education Weakness on Physical Education of College Students, Journal of Jilin Physical Education Institute, 2007, Vol27, p114-115

[9] Deng Hongmei, Wang Bin and Jia Zhenrong, Study Review on Decision Support System of Physical Education Field in Our Country, Journal of Physical Education, 2006, Vol

[10] Wang Ning and Zhao Nan, Discussion on Development Strategy of Urban Physical Education, Postgraduate Dissertation Journal of Shanxi Normal University Physical Culture Institute, 2009, Vol 6, p 9-10 